\title{
Depressão e Ansiedade Gestacionais Relacionadas à Depressão Pós-Parto e o Papel Preventivo do Pré-Natal Psicológico
}

\author{
Gestational Depression and Anxiety Related to Postpartum Depression and the \\ Preventive Role of Psychological Pre-Natal
}

\section{Depresión y Ansiedad Gestacionales Relacionadas à la Depresión Postparto y el Papel Preventivo del Pre-Natal Psicológico}

\author{
Alessandra da Rocha Arrais ${ }^{1}$ \\ Escola Superior de Ciências da Saúde (ESCS) \\ Tereza Cristina Cavalcanti Ferreira de Araujo \\ Universidade de Brasília (UnB) \\ Rafaela de Almeida Schiavo \\ Universidade Paulista (UNIP)
}

\begin{abstract}
Resumo
Introdução: De acordo com a literatura, ansiedade e depressão gestacionais constituem fatores de risco para Depressão Pós-Parto (DPP). Empreendeu-se uma pesquisa-ação visando avaliar a eficácia de um programa denominado Pré-Natal Psicológico (PNP). Realizou-se um estudo experimental de campo, cuja amostra foi composta por 47 gestantes que participaram do PNP (Grupo de Intervenção- GI) e 29 que não participaram (Grupo Controle-GC). Instrumentos: Questionário Gestacional, Inventários Beck de Ansiedade e Depressão e Escala de Depressão Pós-Parto de Edimburgo. Verificou-se alta prevalência $(23,68 \%)$ de risco de DPP. Constatou-se que apenas $10,64 \%$ das gestantes do GI apresentavam risco de desenvolvê-la, em contraposição às mulheres do GC (44,83\%). Em Gl, não se constatou associação entre ansiedade e depressão gestacionais com os sintomas de DPP $(p<0,05)$. Em contrapartida, isto foi observado no GC. Conclusão: o PNP é preventivo ao minimizar o risco desses fatores quanto à DPP.

Palavras-chave: depressão pós-parto, depressão, ansiedade, prevenção, pré-natal psicológico
\end{abstract}

\begin{abstract}
Introduction: According to the literature, gestational anxiety and depression are risk factors for Postpartum Depression- PPD. An action research was undertaken to evaluate the effectiveness of a program called Psychological Pre-Natal (PNP). An experimental field study was carried out in witch the sample consisted of 47 pregnant who participated in the PNP (Intervention Group- GI) and 29 who did not participate (Control Group- GC). Instruments: Gestational Questionnaire, Beck Anxiety and Depression Inventory and Edinburgh Postpartum Depression Scale. There was a high prevalence (23.68\%) of PPD risk. It was verify that only $10.64 \%$ of GI pregnant women presented a risk of developing it, as opposed to the CG women (44.83\%). In GI, there was no association between gestational anxiety and depression with the symptoms of PPD $(p<0.05)$. In contrast, this fact was observed in the GC. Conclusion: the PNP is preventive in minimizing the risk of these factors for PPD.

Keywords: postpartum depression, depression, anxiety, prevention, psychological prenatal care
\end{abstract}

\section{Resumen}

Introducción: Según la literatura, la ansiedad y la depresión gestacional constituyen factores de riesgo para la depresión postparto- DPP. Se emprendió una investigación-acción para evaluar la eficacia de un programa denominado Pre-Natal Psicológico (PNP). Se realizó un estudio experimental de campo donde la muestra fue compuesta por gestantes 47 embarazadas del PNP (Grupo de Intervención- GI) y 29 que no participaron (Grupo Control- GC). Instrumentos: Cuestionario Gestacional, Inventarios Beck de Ansiedad y Depresión y Escala de Depresión Postparto de Edimburgo. Se observó una alta prevalencia $(23,68 \%)$ de riesgo de DPP. Se constató que solo el 10,64\% de las embarazadas del GI presentaban riesgo de desarrollarla, em contraposición a lãs mujeres del GC (44,83\%). En GI, no se constató asociación entre ansiedad y depresión gestacionales con los síntomas de DPP $(p<0,05)$. En cambio, esta asociación fue observada en el GC. Conclusión: el PNP es preventivo al minimizar el riesgo de estos factores de DPP.

Palabras clave: depresión postparto, depresión, ansiedad, prevención, pre-natal psicológico

\footnotetext{
${ }^{1}$ Endereço de contato: SHIS QI 16, conjunto 02, casa 32 . Brasília, DF, Brasil. CEP: 71.640-220. Telefones: +55 $0613244-6947$ e +55 061 99987-7346
} 


\section{Introdução}

Popularmente, ansiedade e depressão seriam sintomas pouco frequentes durante a gravidez, uma vez que essa experiência vital é socialmente percebida como positiva e realizadora para a mulher. Contudo a literatura especializada revela que uma em cada quatro gestantes vivencia tais transtornos, sendo que $48 \%$ daquelas que manifestam ansiedade e $70 \%$ das que apresentam depressão pré-natal permanecem em sofrimento psíquico no período pós-parto e nos primeiros anos de vida de seus filhos, caso não recebam orientação e apoio especializados (Lucci, Otta, David, \& Chelini, 2016).

Cumpre explicitar que a ansiedade gestacional é identificada como um importante fator de risco para Depressão Pós-Parto (DPP). Essa ansiedade atravessa o período gestacional e costuma se estender até o parto, caracterizando-se por um estado de insatisfação, intranquilidade, insegurança, medo, sentimento de incompetência, alterações do sono e tensão muscular que causa dor (Davey, Tough, Adair, \& Benzies, 2011; Figueira, Diniz, \& Silva Filho, 2011; Garfield et al., 2015; Lara, Navarro, Navarrete, \& Le, 2010; Rogers, Kidokoro, Wallendorf, \& Inder, 2013; Santos, Martins, \& Pasqualli, 1999; Simas, Souza, \& Scorsolini-Comin, 2013). Já a depressão é uma doença que afeta principalmente as mulheres em idade fértil e é a principal causa de invalidez em mulheres adultas.

Quando ocorre durante a gestação, é apontada como um dos principais preditores da DPP. Vale também realçar que, nessa época, a prevalência de 'depressão maior' - na qual se evidenciam sintomas psiquiátricos como desânimo, tristeza, anedonia-varia de $10 \%$ a 16\% na população estudada. Com efeito, a DPP é incapacitante, porém não corresponde a um quadro psicótico. Há presença de sintomas psíquicos como desânimo, tristeza, anedonia, que normalmente são minimizados ou ignorados durante as avaliações de pré-natal pela equipe de obstetrícia (Faisal-Cury \& Menezes, 2012; McMahon et al., 2015; Nunes \& Phipps, 2013; Pereira \& Lovisi, 2008; Turkcapar et al., 2015).

Por isso, apesar da alta frequência de queixas depressivas na gravidez, a percepção e o manejo dos sintomas psiquiátricos na gestação estão longe de receber a devida atenção dos ginecologistas e obstetras, o que torna a questão preocupante na medida em que pode haver consequências negativas não só para a mãe, mas também para o bebê (Lucci et al., 2016). A depressão persistente em mulheres grávidas está associada com atraso de desenvolvimento dos filhos aos 18 meses de idade e 4,7 vezes mais risco de terem depressão na adolescência (Jadresic, 2010).

Cabe relembrar que puerpério é um período de intensas mudanças biológicas, psicológicas e sociais, no qual a função de maternagem é agregada a outras condições relacionadas à subjetividade, notadamente a percepção da vulnerabilidade humana. De acordo com a American Psychiatric Association, as estimativas apontavam que, em 2013, 50\% dos casos diagnosticados foram desencadeados quando a mulher ainda estava grávida (American Psychiatric Association [APA], 2014). No cenário internacional, também se verificou que 15\% a $20 \%$ das puérperas são atingidas pela DPP. Em nosso país, as taxas obtidas em algumas pesquisas foram mais elevadas: 32\% e 38\% (Coutinho \& Saraiva, 2008; Theme Filha, Ayers, Gama, \& Leal, 2016).

No entanto menos de $20 \%$ dos cuidados pré-natais visam diagnosticar e tratar dificuldades em nível de Saúde Mental. De fato, estudiosos da área alertam que os sintomas po- 
dem ser minimizados, ou mesmo ignorados, nas avaliações feitas pela equipe de obstetrícia, o que denota pouca articulação entre as ações de saúde para alcance das metas voltadas para a atenção integral. Em outras palavras, ainda que muitas queixas sejam comunicadas de modo claro (ou velado) pela mulher ao longo de seu acompanhamento, o reconhecimento e o manejo clínico dos sintomas não são suficientemente efetivados, o que pode suscitar adversidades para a instauração de uma díade mãe-bebê saudável. Nesse mesmo sentido, alguns estudos indicaram atraso no desenvolvimento de crianças de até 18 meses de idade e mais $4,7 \%$ de chance de apresentar depressão na adolescência do que aqueles nos quais as mães apresentaram esses distúrbios (Jadresic, 2010; Lucci et al., 2016).

Portanto parece indispensável propor, avaliar e comparar serviços e programas destinados ao acompanhamento em Saúde da Mulher e em Saúde Materna e Infantil. De acordo com Arrais e Araújo (2016), no Brasil, programas desenvolvidos pelo Ministério da Saúde para atender essa demanda datam da década de 1980, com a implementação do Programa de Assistência Integral à Saúde da Mulher (PAISM). Esse foi um marco significativo que destacava ações voltadas para os períodos do pré-natal, parto e puerpério. Porém, em função dos seus limites, foi lançado, em 2000, o Programa de Humanização no Pré-Natal e Nascimento (PHPN) com o propósito de oferecer um acompanhamento mais centrado nas necessidades das gestantes. E, posteriormente, foi estabelecida a Rede Cegonha, normatizada pela Portaria no.1.459 de 24 de junho de 2011, com destaque para os direitos humanos e reprodutivos de mulheres, homens, jovens e adolescentes, assim como a qualificação profissional. Observase que, nas unidades de saúde, amplia-se a oferta de serviços em consonância com as diretrizes atuais. Todavia muitas ações ainda privilegiam a dimensão biológica, perpetuando um modelo tradicional de atendimento, em que aspectos psicossociais não são suficientemente considerados e tratados (Arrais \& Araujo, 2016).

Desse ponto de vista, o Pré-Natal Psicológico (PNP) é prática inovadora e complementar ao pré-natal ginecológico, durante a qual se realizam intervenções de natureza psicoprofilática no intuito de propiciar cuidados humanizados durante a gestação. Os temas trabalhados no PNP contemplam: discussão sobre as vias de parto, direito ao acompanhante, distúrbios emocionais do puerpério, ajuda qualificada no pós-parto, o papel dos avós, amamentação, desmistificação da maternidade e paternidade, sexualidade na gravidez.

De modo geral, as atividades propostas pretendem: a) preparar a gestante e sua rede social de apoio para vivenciarem o ciclo gravídico-puerperal; e b) favorecer o desenvolvimento saudável da família tendo em vista a necessidade de elaboração de papéis parentais voltados para a integração de um novo membro, que visa oferecer suporte socioemocional, informacional e instrucional. Esse acompanhamento prevê grupos psicoeducativos sobre gestação, parto e pós-parto. O PNP é um programa aberto, em que a adesão de outras gestantes pode ser feita no decorrer das sessões, e os membros integrantes permanecem pelo tempo desejarem. Com isso, a composição é heterogênea quanto à fase da gestação. Cada sessão semanal dura em torno de três horas e focaliza temas e objetivos específicos, indicados pela equipe ou gerados pelos participantes dos grupos. Pais e avós também são convidados a participar. Adotam-se técnicas variadas de dinâmica de grupo, aulas expositivas e debates, conforme descrito por Arrais e Araujo (2016).

Considerando os pressupostos anteriormente abordados, empreendeu-se uma pesquisa com os seguintes objetivos: a) avaliar os níveis de ansiedade e depressão das gestantes do 
grupo experimental (participantes do PNP) e do grupo controle (não participantes); b) avaliar estas duas subamostras quanto à chance de desenvolver DPP; e c) comparar ambos os grupos e identificar diferenças vinculadas à participação ou não no PNP.

\section{Método}

Este estudo é um recorte do projeto de pesquisa "Fatores de risco e proteção para a depressão pós-parto em gestantes de um hospital público do DF: o papel do pré-natal multiprofissional", aprovado pelo Comitê de Ética Em Pesquisa- FEPECS/SES-DF, sob o Parecer no 979.401. Trata-se de uma pesquisa experimental de campo, onde um grupo foi exposto a uma intervenção chamada de Pré-Natal Psicológico (PNP) e o outro grupo de gestantes que não participaram desta intervenção.

Amostra: Foram incluídas no estudo apenas as gestantes que estavam no 3ำ trimestre da gestação, que ficaram internadas no Setor de Alto Risco (SAR) da maternidade, ao menos um mês, alfabetizadas, residentes do DF e que assinaram ao Termo de Consentimento Livre e Esclarecido (TCLE). A média de gestantes internadas no SAR, no ano de 2015, foi de 86 gestantes ao mês, e o tempo médio de cinco dias. Assim, considerando esses critérios de inclusão e a quantidade média de internações, chegou-se ao total de 76 mulheres, selecionadas por conveniência, para compor a amostra. Destas, 47 participaram do PNP e formaram o Grupo de Intervenção (GI), e 29 não participaram do PNP e compuseram o Grupo Controle (GC).

Local: Maternidade pública de Brasília, considerada referência regional no atendimento à clientela de baixa renda e gestantes de alto risco, no Distrito Federal e entorno.

Instrumentos:

A) Questionário Sociodemográfico: Para caracterização da amostra, foi elaborado um questionário com perguntas fechadas referentes à idade, paridade, estado civil, escolaridade, atividade ocupacional e renda familiar.

B) Inventário de Ansiedade de Beck (BAI): é um questionário de autorrelato com 21 questões de múltipla escolha, utilizada para medir a severidade da ansiedade de um indivíduo. As questões versam sobre como o indivíduo tem se sentido na última semana, expressas em sintomas comuns de ansiedade (como sudorese e sentimentos de angústia). Cada questão apresenta quatro possíveis respostas, e a que se assemelha mais com o estado mental do indivíduo deve ser sinalizada. Os escores alcançados classificarão o nível de ansiedade apresentada: grau mínimo de ansiedade (0-7 pontos), ansiedade leve (8-15 pontos), ansiedade moderada (16-25) e ansiedade severa (26-63 pontos) (Cunha, 2001). Uma revisão da literatura de 1999 concluiu que a BAI é o terceiro instrumento de medida de ansiedade mais utilizado no mundo (Arrais \& Araujo, 2017; Piotrowski, 1999).

C) Inventário de Depressão de Beck - BDI II: esse questionário é desenhado para pacientes acima de 13 anos de idade e é composto por 21 itens sobre como o sujeito tem se sentido nas duas últimas semanas quanto aos sintomas depressivos como desesperança, irritabilidade e culpa ou sentimentos de estar sendo punido, e sintomas físicos como fadiga, perda de peso e diminuição da libido A soma dos escores dos itens individuais fornece um total, que constitui um escore dimensional da intensidade da depressão, que pode ser classificado como: depressão mínima (0-13 pontos), depressão leve (14-19 pontos), depressão modera- 
da (20-28 pontos) e depressão severa (29-63 pontos) (Beck \& Alford, 2014; Cunha, 2001). É um dos instrumentos mais utilizados para medir a severidade da depressão e largamente empregado por profissionais de saúde e pesquisadores em uma variedade de contextos clínicos e de pesquisa (Aliane, Mamede, \& Furtado, 2011; Arrais \& Araujo, 2017).

D) Escala de Depressão Pós-Parto de Edimburgo- EPDS: foi desenvolvida na Grã-Bretanha, por Cox e Holden (2003), para rastrear especificamente sintomas de DPP. Consiste em um instrumento mais utilizado em estudos sobre DPP (Aliane et al., 2011; Arrais \& Araujo, 2017; Galvão, Silva Júnior, Lima, \& Monteiro,2015). É um instrumento validado no Brasil (Cantillino, Zambaldi, Albuquerque, \& Sougey, 2010; Santos et al., 1999), de auto registro, que contém dez questões de sintomas comuns de depressão e utiliza um formato de respostas do tipo likert. O ponto de corte para rastreamento positivo de DPP é pontuação maior ou igual a 12.

Procedimentos de Coleta de dados: Durante o ano de 2015, todas as gestantes que atenderam aos critérios de inclusão, foram convidadas a participar da pesquisa. As que aceitaram o convite e responderam, primeiramente, ao questionário sociodemográfico e aos inventários BAI e BDI II. Em seguida, foram chamadas a participar do grupo de PNP, que ocorreria na própria maternidade, semanalmente, no período vespertino. As sessões do PNP ocorreram, conforme planejamento descrito por Arrais e Araujo (2016), porém, com algumas adaptações, em razão do contexto de enfermaria de alto risco, no qual pacientes "restritas ao leito" não podiam participar de todas as sessões. Cabe explicitar que essa intervenção psicoeducativa prevê abordar e refletir sobre dificuldades, demandas, crenças e mitos sobre a maternidade, especialmente no contexto de uma gestação de alto risco (por exemplo: possibilidade de 'perda' do bebê, malformação fetal, medos associados ao parto prematuro, dores e sofrimento durante o "trabalho de parto". Familiares (por exemplo: pais e avós) foram incluídos nos grupos quando as sessões foram desenvolvidas no horário de visitas à paciente. Após o parto, entre a 4a a 12a semana do puerpério, todas as participantes da amostra responderam a EPDS, durante visitas domiciliares previamente agendadas por telefone.

Procedimentos de análise de dados: os instrumentos foram corrigidos de acordo com o manual de instrução de cada um. Consideraram-se como critério relevante, apenas os casos que fossem classificados com níveis de ansiedade e/ou depressão gestacionais moderado ou grave, ou seja, acima de 16 pontos para o BAl e acima de 18 pontos para o BDI II (Cunha, 2001).

Os dados foram analisados usando o programa estatístico SPSS, versão 21.0 Chicago INc II, USA (Statistical Package for the Social Sciences). Realizou-se estatística descritiva (frequência, porcentagem, média e desvio padrão) para caracterização da amostra, e estatística inferencial para comparação dos grupos por meio do teste Qui-Quadrado $(p<0.05)$. Para a realização do teste Qui-quadrado 2×2, foi necessário binarizar os dados categóricos e numéricos.

\section{Resultados}

Os dados mostraram que, em ambos os grupos intervenção (GI) quanto no controle (GC), o número maior de participantes foi de mulheres com idade acima de 20 anos e primigestas, que em sua maioria vivia com o parceiro, apresentava boa escolaridade tendo ensino médio ou ensino superior, estava empregada, e ganhava até $\mathrm{R} \$ 1.000$. Esses dados podem ser visualizados na tabela 1 , a seguir: 
Tabela 1

Caracterização da amostra $(N=76)$

\begin{tabular}{|c|c|c|c|c|}
\hline \multirow[b]{2}{*}{ Variáveis } & \multicolumn{2}{|c|}{ Grupo de Intervenção } & \multicolumn{2}{|c|}{ Grupo Controle } \\
\hline & Freq. & $\%$ & Freq. & $\%$ \\
\hline \multicolumn{5}{|l|}{ Idade } \\
\hline$<20$ anos & 3 & 6.4 & 3 & 10.3 \\
\hline$>20$ anos & 44 & 93.6 & 26 & 89.7 \\
\hline \multicolumn{5}{|l|}{ Paridade } \\
\hline Primigesta & 27 & 57.4 & 16 & 55.2 \\
\hline Multigesta & 20 & 42.6 & 13 & 44.8 \\
\hline \multicolumn{5}{|l|}{ Estado Civil } \\
\hline Casada & 26 & 55.3 & 13 & 44.8 \\
\hline União Estável & 14 & 29.8 & 6 & 20.8 \\
\hline Solteira & 6 & 12.8 & 6 & 20.7 \\
\hline Divorciada & 1 & 2.1 & 3 & 10.3 \\
\hline Viúva & 0 & 0.0 & 1 & 3.4 \\
\hline \multicolumn{5}{|l|}{ Escolaridade } \\
\hline Ens. Fundamental I & 1 & 2.1 & 2 & 6.9 \\
\hline Ens. Fundamental II & 5 & 10.6 & 3 & 10.3 \\
\hline Ens. Médio & 20 & 42.6 & 10 & 34.5 \\
\hline Ens. Superior & 19 & 40.4 & 13 & 44.8 \\
\hline Não informado & 2 & 4.3 & 1 & 3.4 \\
\hline \multicolumn{5}{|l|}{ Atividade ocupacional } \\
\hline Empregada & 25 & 53.2 & 20 & 69.0 \\
\hline Desempregada & 22 & 46.8 & 9 & 31.0 \\
\hline \multicolumn{5}{|l|}{ Renda familiar } \\
\hline Até $\mathrm{R} \$ 540,00$ & 5 & 10.6 & 3 & 10.3 \\
\hline Até $\mathrm{R} \$ 1000,00$ & 14 & 29.8 & 5 & 17.2 \\
\hline Até $\mathrm{R} \$ 3000,00$ & 6 & 12.8 & 7 & 24.1 \\
\hline$>\mathrm{R} \$ 4000,00$ & 9 & 19.1 & 3 & 10.3 \\
\hline Não informado & 13 & 27.7 & 11 & 37.9 \\
\hline
\end{tabular}

Nota: Freq. Frequência, \% porcentagem.

Observa-se na Tabela 2, que 49,0\% das gestantes do Gl estavam com sintomas de ansiedade, e 25,5\% com sintomas de depressão, porcentagem esta maior do que as grávidas do GC. Infere-se que a incidência desses sintomas nas gestantes do Gl, que trazem sofrimento psíquico, possa ter motivado a participação no PNP. No entanto, no puerpério, há maior prevalência de mulheres com sintomas de DPP nas integrantes do GC, que não participaram do PNP, do que nas do Gl. 
Tabela 2

Indicadores de saúde mental na gestação e nos pós-parto das participantes do Gl (n=47) e GC $(n=29)$

\begin{tabular}{lcccc}
\hline & \multicolumn{2}{c}{ Grupo de Intervenção } & \multicolumn{2}{c}{ Grupo Controle } \\
& Freq. & \% & Freq. & \% \\
\hline Sem Ansiedade & 24 & 51.1 & 19 & 65.5 \\
Com Ansiedade & 23 & 48.9 & 10 & 34.5 \\
Sem depressão & 35 & 74.5 & 23 & 79.3 \\
Com depressão & 12 & 25.5 & 6 & 20.7 \\
Sem DPP & 42 & 89.4 & 16 & 55.2 \\
Com DPP & 5 & 10.6 & 13 & 44.8 \\
\hline
\end{tabular}

Dentre as que participaram do GI, duas gestantes que apresentaram sintomas depressivos no final da gravidez, continuaram a apresentar sintomas depressivos no pós-parto, enquanto que seis do GC continuaram a apresentar sintomas depressivos no pós-parto. Em relação a casos novos de sintomas depressivos no pós-parto, identificou-se que, do Gl, três participantes que não apresentaram sintomas depressivos no final da gravidez, passaram a apresentar no pós-parto, enquanto que sete das que não participaram do PNP passaram a apresentar sintomas depressivos no pós-parto.

A Tabela 3 indica que, ao comparar os dois grupos, nota-se que não houve diferença significativa entre os sintomas de ansiedade e depressão no terceiro trimestre gestacional, entretanto observa-se que há diferença significativa entre os sintomas de depressão pós-parto das que participaram e não participaram do PNP, sendo o GC o que mais apresentou porcentagens de DPP.

Tabela 3

Comparação de sintomas de depressão e ansiedade na gestação e no pós-parto entre o GI $(n=47)$ e o $G C(n=29)$

Saúde Mental

Ansiedade gestacional

Sintomas de depressão gestacional

Sintomas de depressão pós-parto
Comparação entre os grupos Qui-quadrado p-valor

0.217

$0.233 \quad 0.630$

11.598 0.001

\section{Discussão}

A investigação reforça a necessidade apontada em vários estudos como os de Lara et al. (2010), Le, Perry, e Stuart (2011), Bos et al. (2013), Arrais, Lordello, \& Cavados (2015), Galvão, Silva Júnior, Lima, \& Monteiro (2015), e Almeida e Arrais (2016) sobre a importância da identificação dos sintomas iniciais que desencadeiam o quadro patológico no puerpério, pois, quanto antes se detectar os fatores de risco de depressão e ansiedade gestacional, melhor assistência poderá ser oferecida à puérpera. Dessa forma, a alta prevalência de DPP encontrada reforça seu significado como problema de saúde pública, exigindo estratégias 
de prevenção e tratamento, como o PNP (Arrais et al. 2015; Almeida \& Arrais, 2016; Arrais \& Araujo, 2017). O acompanhamento cuidadoso de mães, por meio de ação integrada que considere as variáveis associadas à depressão, pode prevenir graves problemas pessoais e familiares que decorrem da DPP (Galvão et al., 2015).

As características sociodemográficas da amostra não diferiram muito entre Gl e o GC. Esse resultado é semelhante ao encontrado por Figueira et al. (2011) e ao de Spehar e Seidl (2013), pois a composição heterogênea de ambos os grupos se apresenta diversificada quanto ao perfil. Tais características apontam que a maternidade aglutina idades, histórias e motivações diferentes, não é uma experiência igual para todas as mulheres, nem mesmo para a própria mulher em relação à sua própria história de vida. Cada perfil permitirá viver a experiência de forma diferente na construção de sua maternidade (Spehar \& Seidl, 2013).

As mulheres que apresentam sintomas depressivos e ansiosos durante a gravidez têm mais chances de apresentar sintomas de DPP, sobretudo quando não há alguma intervenção psicológica durante a gestação (Davey et al., 2011; Figueira et al., 2011; Garfield et al., 2015; Lara et al., 2010; Rogers, Kidokoro, Wallendorf, \& Inder, 2013). Entretanto os resultados mostraram que a DPP foi mais prevalente justamente no GC composto pelas mulheres que tiveram menos incidência de depressão e ansiedade gestacionais, e não nas do GI, que apresentaram incidência maior desses transtornos.

Todas as mulheres do GC que apresentaram sintomas de depressão na gestação, apresentaram a DPP. Esses achados são concordes como os estudos de Faisal-Cury e Menezes (2012), Nunes e Phipps (2013), Turkcapar et al. (2015), McMahon et al. (2015) e Krob, Godoy, Leite, e Mori (2017), que identificaram a depressão gestacional como um fator de risco para DPP. Ademais, esses dados confirmam a observação clínica já descrita na literatura científica em que algumas mulheres com DPP já estavam deprimidas na gestação (Aliane, Mamede, \& Furtado, 2011), o que justifica a nova nomenclatura de depressão periparto, adotada pela APA a partir de 2014 (APA, 2014). Esses resultados vão ao encontro do estudo realizado por Jadresic (2010). Esse revelou que um terço das gestantes tem sintomas depressivos e / ou ansiedade durante a gravidez, e, se não receberem algum tipo de intervenção psicológica, a prevalência desses transtornos aumenta no pós-parto para mais de 40\%.

Encontrou-se que o grupo intervenção apresentou um percentual maior de gestantes sem DPP. Os resultados para as gestantes que participaram do PNP indicaram que este pode ter sido um fator de proteção para a DPP, pois pesquisas indicam que oferecer atendimento em saúde mental a grávidas pode prevenir sintomas depressivos no pós-parto ou a cronificação dos sintomas presentes na gestação (Bortoletti, 2007; Faisal-Cury, \& Menezes, 2012; McMahon et al., 2015; Nunes, \& Phipps, 2013; Turkcapar et al., 2015)

Pode-se inferir, portanto, que o pré-natal psicológico se revelou como um fator de proteção ao minimizar os riscos que a ansiedade e a depressão gestacional podem causar, sugerindo assim, a eficácia do PNP para prevenir a DPP. Esse resultado sugere que programas de pré-natal com base numa abordagem psicológica, seja ela de referencial teórico prático da psicossomática, seja psicoeducacional, como o PNP, podem contribuir para ampliar fatores de proteção à DPP. Essa inferência é confirmada pelas pesquisas de Kozinszky et al. (2012), Collado, Favrod, Jérôme, \& Hatem (2014), Arrais et al. (2015), Almeida e Arrais (2016) e Krob et al. (2017). 


\section{Conclusão}

Os objetivos deste estudo foram alcançados, uma vez que se avaliaram os níveis de ansiedade e depressão das gestantes da amostra; avaliaram-se as chances das participantes tanto do GI quanto do GC de desenvolver DPP, e compararam-se esses grupos quanto à participação ou não no PNP.

Os resultados encontrados sugerem que ter feito parte do PNP diminuiu a chance das participantes do GI desenvolverem a DPP, apesar de terem os fatores de risco de depressão e ansiedade mais graves e mais presentes na gestação. Isso mostra que o PNP, atrelado ao pré-natal obstétrico e aliado a outros fatores de proteção, que devem ser investigados em pesquisas futuras, pode minimizar os efeitos dos fatores de risco para DPP.

O PNP revelou-se como uma ferramenta tecnológica eficaz, de baixo custo, para prevenção da DPP, que pode ser implementada por equipes multidisciplinares, em hospitais públicos e privados, clínicas da mulher e outros contextos assistenciais. Em suma, a assistência psicológica na gestação, por meio da utilização do PNP, é um importante instrumento psicoprofilático que deveria ser implementado em postos de saúde e serviços de pré-natal no Brasil. Compete mencionar que esta pesquisa converge com as atuais preocupações sociopolíticas nacionais, quem vêm sendo discutidas no âmbito legislativo, uma vez que a Comissão de Constituição e Justiça e de Cidadania (CCJ), da Câmara dos Deputados, aprovou em 06/06/18 o Projeto de Lei 702/15², que prevê que gestantes e mães de recém-nascidos recebam acompanhamentos psicológico, incluindo-se a avaliação para detecção da DPP.

\section{Informação sobre Apoio Financeiro e Institucional}

Essa pesquisa recebeu o apoio financeiro e institucional da Fundação de Apoio à Pesquisa do Distrito Federal (FAP-DF), por meio do Edital 10/2010- Demanda induzida: Área ciências da saúde. № do processo: 193.000.393/2010. Afirmamos que não houve conflito de interesses entre a pesquisa e o apoio recebido.

\section{Referências}

Aliane, P. P., Mamede, M. V., \& Furtado, E. F. (2011). Revisão sistemática sobre fatores de risco associados à depressão pós-parto. Psicologia em Pesquisa, 5(2), 146-155. Disponível em http://periodicos.ufjf.br/index.php/psicologiaempesquisa/article/view/23598

Almeida, N. M. C., \& Arrais, A. R. (2016). O Pré-Natal Psicológico como Programa de Prevenção à Depressão Pós-Parto. Psicologia: Ciência e Profissão, 36(4), 847-863. doi: 10.1590/1982-3703001382014

American Psychiatric Association (2014). Diagnostic and statistical manual of mental disorders - DSM-5 (5a ed.). Washington, DC: American Psychiatric Association. doi: 10.1176/appi. books.9780890425596

Arrais, A. R., Lordello, S. R., \& Cavados, G. C. F. (2015). O pré-natal psicológico como fator de proteção à depressão pós-parto. In S. G. Murta, C. França, L. K. B. Santos, \& L. Polejack (Eds.), Prevenção e promoção em saúde mental: Fundamentos, planejamento e estratégias de intervenção (pp. 601-621). Novo Hamburgo, RS: Sinopsys.

$\overline{{ }^{2} \text { http://www.camara.gov.br/proposicoesWeb/fichadetramitacao?idProposicao=996806 }}$ 
Arrais, A. R., \& Araujo, T. C. C. F. (2016). Pré-natal psicológico: perspectivas para atuação do psicólogo em saúde materna no Brasil. Revista da Sociedade Brasileira de Psicologia Hospitalar, 19(1), 103-116. Disponível em http://pepsic.bvsalud.org/scielo. php?script=sci_arttext\&pid=S1516-08582016000100007

Arrais, A. R., \& Araujo, T. C. C. F. (2017). Depressão pós-parto: uma revisão sobre fatores de risco e de proteção. Psicologia, Saúde \& Doenças, 18(3), 828-845 doi: 10.15309/17psd180316

Beck, A. T., \& Alford, B. A. (2014). Depression: Causes and treatment (2a ed.). Philadelphia: University of Pennsylvania Press.

Bortoletti, F. F. (2007). Psicodinâmica do ciclo gravídico puerperal. In F. F. Bortoletti (Ed.), Psicologia na prática obstétrica: Abordagem interdisciplinar (pp. 21-31). Barueri, SP: Manole.

Bos, S. C., Macedo, A., Marques, M., Pereira, A. T., Maia, B. R., Soares, M. J., . . A Azevedo, M. $H$. (2013). Is positive affect in pregnancy protective of postpartum depression? Revista Brasileira de Psiquiatria, 35, 5-12. doi: 10.1016/j.rbp.2011.11.002

Cantillino, A., Zambaldi, C. F., Albuquerque, T. L. C., \& Sougey, E. B. (2010). Depressão pósparto em Recife - Brasil: Prevalência e associação com fatores biossociodemográficos. Jornal Brasileiro de Psiquiatria, 59, 1-9. doi: 10.1590/S0047-20852010000100001

Collado, M. A. O., Favrod, M. S., Jérôme, F., \& Hatem, M. (2014). Antenatal psychosomatic programming to reduce postpartum depression risk and improve childbirth outcomes: $\mathrm{A}$ randomized controlled trial in Spain and France. BioMed Central Pregnancy \& Childbirth, 14, 1-12. doi: 10.1186/1471-2393-14-22

Coutinho, M. P. L., \& Saraiva, E. R. A. (2008). O sofrimento psíquico no puerpério: um estudo psicológico. Revista Mal-Estar e Subjetividade, 81(2), 505-527. Disponível em http://www. revispsi.uerj.br/v8n3/artigos/html/v8n3a14.html

Cox, J., \& Holden, J. (2003). Perinatal mental health: A guide to the Edinburgh Postnatal Depression Scale (EPDS). London: Royal College of Psychiatrists, Gaskell.

Cunha, J. A. (2001). Manual da versão em português das Escalas Beck. São Paulo: Casa do Psicólogo.

Davey, H. L., Tough, S. C., Adair, C. E., \& Benzies, K. M. (2011). Risk factors for subclinical and major postpartum depression among a community cohort of canadian women. Maternal Child Health Journal, 15(7), 866-875. doi: 10.1007/s10995-008-0314-8

Faisal-Cury, A., \& Menezes, P. R. (2012). Depressão antenatal prediz fortemente depressão pós-parto na atenção básica à saúde. Revista Brasileira de Psiquiatria, 34, 446-450. doi: 10.1016/j.rbp.2012.01.003

Figueira, P. G., Diniz, L. M., \& Silva Filho, H. C. (2011). Características demográficas e psicossociais associadas à depressão pós-parto em uma amostra de Belo Horizonte. Revista de Psiquiatria do Rio Grande do Sul, 33(2), 71-75. doi: 10.1590/S0101-81082011005000009

Galvão, A. C. C., Silva Júnior, F. J. G., Lima, L. A. A, \& Monteiro, C. F. S. (2015). Prevalência de depressão pós-parto e fatores associados: Revisão sistemática. Revista On Facema, 1, 54 58. Disponível em http://www.facema.edu.br/ojs/index.php/ReOnFacema/article/view/3 Garfield, L., Holditch-Davis, D., Carter, C. S., McFarlin, B. L., Schwertz, D., Seng, J. S., . . . White-Traut, R. (2015). Risk factors for postpartum depressive symptoms in low-income women with very low-birth-weight infants. Advances in Neonatal Care Journal, 15, 3-8. doi: 10.1097/ANC.0000000000000131 
Jadresic, M., E. (2010). Depresion en el embarazo y el puerpério. Revista Chilena de Neuropsiquiatría, 48(4), 269-278. doi: 10.4067/S0717-92272010000500003

Krob, A., Godoy, J., Leite, K., \& Mori, S. (2017). Depressão na gestação e no pós-parto e a responsividade materna nesse contexto. Revista Psicologia e Saúde, 9(3), 3-16. doi: 10.20435/pssa.v9i3.565

Lara, M. A., Navarro, C., Navarrete, L., \& Le, H.-N. (2010). Tasas de retención y variables que la predicen em um estúdio aleatorio controlado longitudinal para prevenir la depresión posparto. Salud Mental, 33, 429-436. Disponível em http://www.scielo.org.mx/scielo. php?script=sci_arttext \&pid=S0185-33252010000500007

Le, H.-N., Perry, D. F., \& Stuart, E. A. (2011). Randomized controlled trial of a preventive intervention for perinatal depression in high-risk latinas. Journal of Consulting and Clinical Psychology, 79, 135-141. doi: 10.1037/a0022492

Lucci, T. K., Otta, E., David, V. F., \& Chelini, M. O. M. (2016). Depressão materna e concentração de cortisol de recém-nascidos em uma amostra brasileira. Revista Psico, 47(2), 140-147. doi: 10.15448/1980-8623.2016.2.23655

Kozinszky, Z. Dudas, R. B., Devosa, I., Csatordai, S., Tóth, E., Szabó, D., . . Pál, A. (2012). Can a brief antepartum preventive group intervention help reduce postpartum depressive symptomatology? Psychotherapy and Psychosomatics, 81, 98-107. doi: 10.1159/000330035

McMahon, C. A., Boivin, J., Gibson, F. L., Hammarberg, K., Wynter, K., \& Fisher, J. R. (2015). Older maternal age and major depressive episodes in the first two years after birth: Findings from the parental age and transition to parenthood Australia (PATPA) study. Journal of Affective Disorders, 175, 454-462. doi: 10.1016/j.jad.2015.01.025

Nunes, A. P., \& Phipps, M. G. (2013). Postpartum depression in adolescent and adult mothers: comparing prenatal risk factors and predictive models. Maternal and Child Health Journal, 17, 1071-1079. doi: 10.1007/s10995-012-1089-5

Pereira, P. K., \& Lovisi, G. M. (2008). Prevalência da depressão gestacional e fatores associados. Revista de Psiquiatria Clínica, 35, 144-153. Disponível em http://www.scielo.br/pdf/rpc/ v35n4/04.pdf

Piotrowski, C. (1999). The status of the Beck Anxiety Inventory in contemporary research. Psychological Reports, 85(1), 261-262. doi: 10.2466/pr0.1999.85.1.261

Rogers, C. E., Kidokoro, H., Wallendorf, M., \& Inder, T. E. (2013). Identifying mothers of very preterm infants at-risk for postpartum depression and anxiety prior to discharge. Journal of Perinatology, 33, 171-176. doi: http://dx.doi.org/10.1038/jp.2012..7

Santos, M. F. S., Martins, F. C., \& Pasquali, L. (1999). Escala de auto-avaliação de DPP: estudo no Brasil. Revista de Psiquiatria Clínica, 26(2), 90-95.

Simas, F. B., Souza, L. V., \& Scorsolini-Comin, F. (2013). Significados da gravidez e da maternidade: Discursos de primíparas e multíparas. Revista Psicologia: Teoria e Prática, 15(1), 19-34. Disponível em http://www.redalyc.org/articulo.oa?id=193826310013

Spehar, M. C., \& Seidl, E. M. F. (2013). Percepções maternas no método canguru: contato pele a pele, amamentação e autoeficácia. Psicologia em Estudo, 18, 647-656. Disponível em http://www.scielo.br/pdf/pe/v18n4/07.pdf

Theme Filha, M. M., Ayers S., Gama S. G., \& Leal, M. C. (2016). Factors associated with postpartum depressive symptomatology in Brazil: The birth in Brazil national research 
study, 2011/2012. Journal of Affective Disorders, 194, 159-167. doi: 10.1016/j. jad.2016.01.020

Turkcapar, A. F., Kadıoğlu, N., Aslan, E., Tunc, S., Zayıfoğlu, M., \& Mollamahmutoğlu, L. (2015). Sociodemographic and clinical features of postpartum depression among Turkish women: a prospective study. BioMed Central Pregnancy \& Childbirth, 15, 108. doi: 10.1186/ s12884-015-0532-1

Recebido: $27 / 02 / 2018$

Última revisão: 05/07/2018

Aceite final: 16/08/2018

\section{Sobre as autoras:}

Alessandra da Rocha Arrais - Pós-Doutora e Doutora pelo Programa de Pós-graduação em Psicologia Clínica e Cultura do Departamento de Psicologia da Universidade de Brasília (UnB), Brasília. Docente permanente do Programa de Mestrado Profissional em Ciências para a Saúde da Escola Superior de Ciências da Saúde (ESCS), Brasília. E-mail: alearrais@gmail.com, Orcid: http://orcid.org/0000-0002-1057-6914

Tereza Cristina Cavalcanti Ferreira de Araujo - Pós-Doutora pela Unesco, França. Doutora pela Université de Paris X - Nanterre. Professora do Departamento de Psicologia Clínica da UnB, Orientadora de Mestrado e de Doutorado no Programa de Psicologia Clínica e Cultura e no Programa de Bioética da UnB, Brasília. E-mail: araujotc@unb.br, Orcid: http://orcid.org/0000-0002-5204-8124

Rafaela de Almeida Schiavo - Doutora pela Faculdade de Medicina da Universidade Estadual Paulista (UNESP/Botucatu), São Paulo. Docente nos cursos de Psicologia, Pedagogia e Engenharia da Universidade Paulista (UNIP/Bauru) campus Bauru e Docente no Instituto Municipal de Ensino Superior de São Manuel (IMESSM), São Paulo. E-mail: rafaela.schiavo@gmail.com, Orcid: http://orcid.org/0000-0001-7290-3610 